Original article

\title{
Predicting critical illness on initial diagnosis of COVID-19 based on easily obtained clinical variables: development and validation of the PRIORITY model
}

\author{
Miguel Martínez-Lacalzada ${ }^{1, \dagger}$, Adrián Viteri-Noël ${ }^{1, \dagger}$, Luis Manzano ${ }^{1,2, *}$, \\ Martin Fabregate ${ }^{1}$, Manuel Rubio-Rivas ${ }^{3}$, Sara Luis García ${ }^{4}$, \\ Francisco Arnalich-Fernández ${ }^{5}$, José Luis Beato-Pérez ${ }^{6}$, Juan Antonio Vargas-Núñez ${ }^{7}$, \\ Elpidio Calvo-Manuel ${ }^{8}$, Alexia Constanza Espiño-Álvarez ${ }^{9}$, Santiago J. Freire-Castro ${ }^{10}$, \\ Jose Loureiro-Amigo ${ }^{11}$, Paula Maria Pesqueira Fontan ${ }^{12}$, Adela Pina ${ }^{13}$, \\ Ana María Álvarez Suárez ${ }^{14}$, Andrea Silva-Asiain ${ }^{15}$, Beatriz García-López ${ }^{16}$, \\ Jairo Luque del Pino ${ }^{17}$, Jaime Sanz-Cánovas ${ }^{18}$, Paloma Chazarra-Pérez ${ }^{19}$, \\ Gema María García-García ${ }^{20}$, Jesús Millán Núñez-Cortés ${ }^{4}$, José Manuel Casas-Rojo ${ }^{21}$, \\ Ricardo Gómez-Huelgas ${ }^{18}$, for the SEMI-COVID-19 Network ${ }^{\ddagger}$
}

1) Internal Medicine Department, Hospital Universitario Ramón y Cajal, IRYCIS, Madrid, Spain

2) Faculty of Medicine, Universidad de Alcalá (UAH), Alcalá de Henares, Madrid, Spain

3) Internal Medicine Department, Bellvitge University Hospital-IDIBELL, L'Hospitalet de Llobregat, Barcelona, Spain

4) Internal Medicine Department, Gregorio Marañon University Hospital, Madrid, Spain

5) Internal Medicine Department, La Paz University Hospital, Madrid, Spain

6) Internal Medicine Department, Albacete University Hospital Complex, Albacete, Spain

7) Internal Medicine Department, Puerta de Hierro University Hospital, Majadahonda, Madrid, Spain

${ }^{8)}$ Internal Medicine Department, San Carlos Clinical Hospital, Madrid, Spain

9) Internal Medicine Department, La Princesa University Hospital, Madrid, Spain

10) Internal Medicine Department, A Coruña University Hospital, A Coruña, Spain

11) Internal Medicine Department, Moisès Broggi Hospital, Sant Joan Despí, Barcelona, Spain

12) Internal Medicine Department, Santiago Clinical Hospital, Santiago de Compostela, A Coruña, Spain

13) Internal Medicine Department, Dr Peset University Hospital, University of Valencia, Valencia, Spain

14) Internal Medicine Department, Cabueñes Hospital, Gijón, Asturias, Spain

15) Internal Medicine Department, Nuestra Señora Del Prado Hospital, Talavera de la Reina, Toledo, Spain

16) Internal Medicine Department, Zamora Hospital Complex, Zamora, Spain

17) Internal Medicine Department, Costa Del Sol Hospital, Marbella, Málaga, Spain

18) Internal Medicine Department, Regional University Hospital of Málaga, Biomedical Research Institute of Málaga (IBIMA), University of Málaga (UMA), Málaga, Spain

19) General Internal Medicine Department, San Juan de Alicante University Hospital, San Juan de Alicante, Alicante, Spain

20) Internal Medicine Department, Badajoz University Hospital Complex, Badajoz, Spain

21) Internal Medicine Department, Infanta Cristina University Hospital, Parla, Madrid, Spain

\section{A R T I C L E I N F O}

Article history:

Received 5 March 2021

Received in revised form

2 July 2021

Accepted 3 July 2021

Available online 15 July 2021

Editor: M. Paul

\section{A B S T R A C T}

Objectives: We aimed to develop and validate a prediction model, based on clinical history and examination findings on initial diagnosis of coronavirus disease 2019 (COVID-19), to identify patients at risk of critical outcomes.

Methods: We used data from the SEMI-COVID-19 Registry, a cohort of consecutive patients hospitalized for COVID-19 from 132 centres in Spain (23rd March to 21st May 2020). For the development cohort, tertiary referral hospitals were selected, while the validation cohort included smaller hospitals. The primary outcome was a composite of in-hospital death, mechanical ventilation, or admission to intensive

\footnotetext{
* Corresponding author. Luis Manzano, Servicio de Medicina Interna, Hospital Universitario Ramón y Cajal, Ctra de Colmenar Viejo, km 9,100, 28034, Madrid, Spain. E-mail address: luis.manzano@uah.es (L. Manzano).

† M. Martínez-Lacalzada and A. Viteri-Noël contributed equally.

$\ddagger$ The members of SEMI-COVID-19 Network are listed at Supplementary Appendix S1
} 
Keywords:

COVID-19

Critical illness

Easily obtained clinical variables

Initial assessment

Prognostic models care unit. Clinical signs and symptoms, demographics, and medical history ascertained at presentation were screened using least absolute shrinkage and selection operator, and logistic regression was used to construct the predictive model.

Results: There were 10433 patients, 7850 in the development cohort (primary outcome 25.1\%, 1967/ 7850 ) and 2583 in the validation cohort (outcome 27.0\%, 698/2583). The PRIORITY model included: age, dependency, cardiovascular disease, chronic kidney disease, dyspnoea, tachypnoea, confusion, systolic blood pressure, and $\mathrm{SpO}_{2} \leq 93 \%$ or oxygen requirement. The model showed high discrimination for critical illness in both the development (C-statistic 0.823; 95\% confidence interval $(\mathrm{CI}) 0.813,0.834)$ and validation (C-statistic $0.794 ; 95 \%$ CI $0.775,0.813$ ) cohorts. A freely available web-based calculator was developed based on this model (https://www.evidencio.com/models/show/2344).

Conclusions: The PRIORITY model, based on easily obtained clinical information, had good discrimination and generalizability for identifying COVID-19 patients at risk of critical outcomes. Miguel MartínezLacalzada, Clin Microbiol Infect 2021;27:1838

(C) 2021 The Authors. Published by Elsevier Ltd on behalf of European Society of Clinical Microbiology and Infectious Diseases. This is an open access article under the CC BY-NC-ND license (http:// creativecommons.org/licenses/by-nc-nd/4.0/).

\section{Introduction}

Coronavirus disease 2019 (COVID-19) has spread globally, with a clinical spectrum ranging from an asymptomatic state to critical illness [1-3]. Notably, Spain was one of the countries with the highest incidence of COVID-19 during the first pandemic peak [4]. To optimize the use of limited healthcare resources, it is essential to identify, as early as possible, those patients who are at high risk of progressing to critical illness.

To date, studies of COVID-19 prognostic factors have focused on laboratory measurements and radiological examinations obtained following admission [5-15], which are not available in outpatient or resource-limited settings. Recently published well-developed models tend not to include clinical variables obtained from history and examination carried out on initial assessment [9-13]. Where one machine-learning model has addressed basic clinical features, it has narrowed down the prediction to the mortality outcome only and lacks wider generalizability [16]. Furthermore, a critical appraisal of the COVID-19 models has shown poor reporting and high risk of bias [14].

Prediction models based on easy-to-collect data have previously been developed for other infectious diseases, e.g. meningitis and pneumonia [17-19]. As a global health emergency, management of COVID-19 would benefit from a prediction model that could be readily applied for initial diagnosis. Therefore, we developed and externally validated a prediction model-based on easily obtainable clinical measures at presentation with confirmed COVID-19 diagnosis-to identify patients at risk of developing critical outcomes.

\section{Methods}

\section{Study design and data source}

This study was based on the SEMI (Sociedad Española de Medicina Interna) COVID-19 Registry [20]. It is an ongoing multicentre nationwide cohort of consecutive patients hospitalized for COVID-19 across Spain. Eligibility criteria were age $\geq 18$ years, confirmed diagnosis of COVID-19 (defined as a positive result on real-time reverse-transcription-PCR (RT-PCR) for the presence of severe acute respiratory syndrome coronavirus 2 (SARS-CoV-2) in nasopharyngeal swab specimens or sputum samples), first hospital admission for COVID-19, and hospital discharge or in-hospital death [20]. The SEMI-COVID-19 Registry was approved by the Provincial Research Ethics Committee of Málaga (Spain) and the
Institutional Research Ethics Committees of each participating hospital.

For the study, we retrieved from the Registry clinical baseline data, history of previous medication, and comorbidities collected on admission, as well as complications during hospitalization and status at discharge. We used data from patients admitted to 132 hospitals between 23rd March and 21st May 2020. We chose hospital complexity as the criterion to assess the transportability of the prognostic model in a setting other than the one in which it was derived $[21,22]$. Patients admitted to tertiary referral hospitals ( $\geq 300$ beds, according to the Ministry of Health of Spain [23]) were selected for the development cohort, while patients from smaller hospitals ( $<300$ beds) were included in a separate validation cohort.

\section{Outcome description}

The primary outcome, critical illness during hospitalization, was defined as the composite of in-hospital death, mechanical ventilation or admission to the intensive care unit (ICU), according to previously published studies [10,24,25].

\section{Potential predictors}

To develop a predictive model based only on easily measurable variables registered at admission, we considered clinical signs and symptoms, demographic variables, and medical history. An initial list of 29 candidate variables was selected based on review of the existing evidence [5-16], clinical plausibility, and relevance to clinical care. To improve consensus on model applicability, a oneround online questionnaire was conducted among a multidisciplinary panel of 24 physicians involved in the clinical management of COVID-19 at nursing homes, emergency departments, primary care centres and hospitalization wards (six per each setting). The panellists were asked to rate (on a nine-point Likert scale) the availability/reliability of each predictor, its ability to predict the outcome, the best way to merge predictors of rare occurrence, and the maximum number of variables the model should contain. Agreement was considered when seven or fewer panellists rated outside the three-point region containing the median [26].

\section{Statistical analysis}

The predictive model, called PRIORITY, was presented as the formula for estimating the probability of COVID-19 critical illness 
outcome, as well as an associated web-based calculator. Patients' characteristics were summarized as frequencies and percentages or means and standard deviations. Statistical analysis was performed using R v4.0.0, with mice, mfp, glmnet, pROC, rms and rmda packages.

\section{Model development}

Missing values in the potential predictors were imputed using single imputation, a reasonable alternative to multiple imputation when dealing with relatively few missing values [27]. A stochastic single imputation dataset was created for both cohorts (development and validation) through multiple imputation by chained equations. Quantitative variables were kept as continuous to avoid loss of prognostic information, and non-linear relationships were modelled by multivariate fractional polynomials with a maximum of two degrees of freedom [28]. The least absolute shrinkage and selection operator (LASSO) was the feature selection method used to reduce the number of predictors down to the maximum agreed by the expert panel [29]. Briefly, the potential predictors were entered into the LASSO regularization process, which penalizes the coefficients by gradually shrinking them to zero. We selected the penalty parameter $(\lambda)$ that minimized the deviance within the given maximum number of predictors. Those variables with nonzero coefficients were retained for risk estimation using a logistic regression model. Coefficients were presented as odds ratios (ORs) and $95 \%$ confidence intervals (95\%CIs).

\section{Model performance}

Nagelkerke's $\mathrm{R}^{2}$ and Brier score were used as overall performance measures. We assessed the discriminative ability of the model using the C-statistic, calculated as the area under the receiver operating characteristic curve, with $95 \% \mathrm{CI}$. Calibration of the model was visually assessed by plotting deciles of predicted versus observed probabilities, and the calibration slope with $95 \% \mathrm{CI}$ was calculated [22].

\section{Model validation}

Internal validation was carried out to assess optimism-corrected performance by repeating the entire model development over 1000 bootstrap samples drawn from the development cohort [27]. We externally validated the model in a separate cohort of patients admitted at smaller hospitals to evaluate model transportability [21]. Within this validation cohort, we reassessed model performance and compared its discrimination ability with models based on oxygen saturation and/or age, the most discriminating univariate predictors for in-hospital mortality previously reported [15]. We also undertook a decision curve analysis, a method to ascertain the adequacy of prediction models based on the relative value of benefits (true positives) and harms (false positives) [30]. We plotted the net benefit of the models for the full range of critical illness probability thresholds.

\section{Sensitivity analysis}

To assess the impact of assumptions adopted in the model development, we carried out a complete-case analysis using only those patients with complete data in the potential predictors. We also developed models without restricting the maximum number of predictors ( $\lambda$ at one-standard-error of the minimum) or using linear continuous predictors instead of non-linear terms.

\section{Results}

We considered data from 10433 patients included in the SEMICOVID-19 Registry. The development cohort included 7850 patients, of whom 1967 (25.1\%) presented critical illness: 650 (8.3\%) were admitted to the ICU and 1598 (20.4\%) died. The mean age was $65.8 \pm 16.4$ years and 57.2\% (4483/7834) were male. Demographics and clinical characteristics for the development cohort are shown on Table 1.

\section{Model development and performance}

From an initial list of 29 candidate variables, the expert panel forged an agreement on 21 potential predictors for further evaluation in the predictive model. Chronic liver disease, previous medication with angiotensin converting enzyme inhibitors or angiotensin receptor blockers, cough, arthralgia/myalgia, ageusia/ anosmia, asthenia/anorexia, headache, gastrointestinal symptoms were excluded. Consensus was achieved for including a range between five and nine variables in the final model. For transparency, univariate analysis is shown in Supplementary Material Table S1, even though it was not part of the model development process. The 21 potential predictors were included in the LASSO selection process, retaining a subset of nine variables as the best predictors of critical illness (Supplementary Material Fig. S1). A multivariable logistic regression model was then fitted with these nine variables. All of them, except for moderate or severe dependency, were statistically significant (Table 2).

Based on the logistic regression model, the probability of critical COVID-19 illness could be calculated as:

Probability $(\%)=100 /(1+\exp (-\mathrm{z}))$, where $\mathrm{z}=$ $-4.665+2.663 \cdot\left((\text { age } / 100)^{2}\right)+0.164 \cdot($ dependency $)+0.316$. (cardiovascular disease) $+0.586 \cdot($ chronic kidney disease) $+0.504 \cdot($ dyspnoea $)+0.844 \cdot\left(1 /(\mathrm{SBP} / 100)^{2}\right)+0.911 \cdot($ tachypnoea $)$ $+1.200 \cdot\left(\mathrm{SpO}_{2} \leq 93 \%\right.$ or oxygen requirement $)+0.681 \cdot($ confusion $)$.

All predictors were coded as binary variables ( 1 when present and 0 when absent) except for age (years) and systolic blood pressure (SBP, $\mathrm{mmHg}$ ). We also developed an online calculator based on this model (Supplementary Material Fig. S2), accessible at https://www.evidencio.com/models/show/2344.

In the development cohort, the PRIORITY model had an $\mathrm{R}^{2}$ of 0.347 and a Brier score of 0.138 . The apparent C-statistic was 0.823 (95\%CI 0.813, 0.834) (Fig. 1a). After bootstrap internal validation, optimism-corrected C-statistic was $0.821(95 \% \mathrm{CI}$ $0.810,0.832)$. The model showed good calibration across the range of predicted probabilities within the development cohort (calibration slope 0.996, 95\%CI 0.989, 0.999; Supplementary Material Fig. S3a).

\section{External validation}

The validation cohort included 2583 patients, of whom 698 (27.0\%) presented critical illness: 200 (7.7\%) were admitted to the ICU and 594 (23.0\%) died. The mean age was $69.5 \pm 16.0$ years, $54.8 \%$ (1415/2580) were male (Table 1). The PRIORITY model showed good discrimination for critical illness within the validation cohort (C-statistic 0.794, 95\%CI 0.775, 0.813) (Fig. 1b), and a calibration slope of $0.875,95 \% \mathrm{CI} 0.790,0.960$ (Supplementary Material Fig. S3b).

Our model compared well against the risk stratification based on univariate models including age (C-statistic $0.707,95 \% \mathrm{CI} 0.686$, 0.729 ) or $\mathrm{SpO}_{2} \leq 93 \% /$ oxygen requirement at admission (C-statistic $0.652,95 \%$ CI $0.635,0.670$ ) as sole predictors. Likewise, the PRIORITY model had better discriminative ability than the model including both age and $\mathrm{SpO}_{2} \leq 93 \%$ /oxygen supply (C-statistic $0.751,95 \% \mathrm{CI}$ $0.731,0.771$ ). Additionally, decision curve analysis showed that the PRIORITY model had higher net benefit across a wide range of threshold probabilities for developing critical illness compared to risk stratification using age and/or $\mathrm{SpO}_{2} \leq 93 \%$ /oxygen supply (Fig. 2). 
Table 1

Demographic and clinical characteristics among patients included in the development and validation cohorts

\begin{tabular}{|c|c|c|c|c|c|}
\hline & & \multicolumn{2}{|l|}{ Development cohort } & \multicolumn{2}{|l|}{ Validation cohort } \\
\hline & & No of patients $(\%)$ or mean \pm SD & Total No (\%) & No of patients $(\%)$ or mean \pm SD & Total No (\%) \\
\hline \multicolumn{6}{|c|}{ Characteristics of the population } \\
\hline Critical illness & & 1967 (25.1\%) & $7850(100 \%)$ & $698(27.0 \%)$ & $2583(100 \%)$ \\
\hline Age [years] & & $65.8 \pm 16.4$ & $7816(99.6 \%)$ & $69.5 \pm 16.0$ & $2575(97.3 \%)$ \\
\hline Male & & $4483(57.2 \%)$ & $7834(99.8 \%)$ & $1415(54.8 \%)$ & $2580(99.9 \%)$ \\
\hline \multirow[t]{3}{*}{ Ethnicity } & Caucasian & $6836(89.1 \%)$ & 7677 (98.8\%) & $2340(91.0 \%)$ & $2572(99.6 \%)$ \\
\hline & Latino & $693(9.0 \%)$ & & $193(7.5 \%)$ & \\
\hline & Other & $148(1.9 \%)$ & & $39(1.5 \%)$ & \\
\hline \multirow[t]{3}{*}{ Smoking history } & Never & $5270(70.9 \%)$ & $7433(94.7 \%)$ & $1625(65.7 \%)$ & $2475(95.8 \%)$ \\
\hline & Former smoker & $1764(23.7 \%)$ & & $718(29.0 \%)$ & \\
\hline & Active Smoker & $399(5.4 \%)$ & & $139(5.3 \%)$ & \\
\hline \multicolumn{6}{|l|}{ Medical history } \\
\hline Obesity $^{\mathrm{a}}$ & & $1665(23.7 \%)$ & $7012(89.3 \%)$ & $584(24.3 \%)$ & $2401(93.0 \%)$ \\
\hline Hypertension & & $3803(48.6 \%)$ & $7833(99.8 \%)$ & $1444(56.1 \%)$ & $2576(99.7 \%)$ \\
\hline Diabetes mellitus & & $1440(18.4 \%)$ & $7820(99.6 \%)$ & $509(19.8 \%)$ & $2570(99.5 \%)$ \\
\hline Cardiovascular dis & & $1974(25.3 \%)$ & $7800(99.4 \%)$ & $806(31.7 \%)$ & $2545(98.5 \%)$ \\
\hline Pulmonary diseas & & $1625(20.9 \%)$ & $7776(99.1 \%)$ & $576(22.6 \%)$ & $2583(98.9 \%)$ \\
\hline Severe chronic kid & & $488(6.2 \%)$ & $7825(99.7 \%)$ & $163(6.3 \%)$ & $2583(99.7 \%)$ \\
\hline Malignancy $^{\mathrm{e}}$ & & $793(10.2 \%)$ & $7803(99.4 \%)$ & $259(10.1 \%)$ & $2571(99.5 \%)$ \\
\hline Immunocomprom & & $650(8.6 \%)$ & $7549(96.2 \%)$ & $187(7.6 \%)$ & $2473(95.7 \%)$ \\
\hline Dependency (mod & & $1129(14.7 \%)$ & $7701(98.1 \%)$ & $605(23.7 \%)$ & 2555 (98.9\%) \\
\hline \multicolumn{6}{|c|}{ Symptoms at admission } \\
\hline Fever $^{\mathrm{h}}$ & & $5138(67.0 \%)$ & $7663(97.6 \%)$ & $1670(65.6 \%)$ & $2544(98.5 \%)$ \\
\hline Dyspnoea & & $4427(56.7 \%)$ & $7805(99.4 \%)$ & $1523(59.4 \%)$ & $2562(99.2 \%)$ \\
\hline \multicolumn{6}{|c|}{ Clinical signs and physical exploration at admission } \\
\hline $\mathrm{SBP}(\mathrm{mmHg})$ & & $129.0 \pm 21.5$ & $7430(94.6 \%)$ & $127.6 \pm 21.0$ & $2451(94.9 \%)$ \\
\hline HR (beats/minute) & & $88.6 \pm 17.4$ & $7500(95.5 \%)$ & $87.5 \pm 17.5$ & $2504(96.9 \%)$ \\
\hline Tachypnoea $(>20$ & & $2271(29.9 \%)$ & $7604(96.9 \%)$ & $879(35.1 \%)$ & $2504(96.9 \%)$ \\
\hline $\mathrm{SpO}_{2} \leq 93 \%$ or oxy & ment at presentation & $4152(52.9 \%)$ & 7842 (99.9\%) & $1605(62.1 \%)$ & $2583(100 \%)$ \\
\hline Pulmonary rales & & $4630(60.7 \%)$ & $7626(97.1 \%)$ & $1588(63.6 \%)$ & $2495(96.6 \%)$ \\
\hline Confusion & & $849(11.0 \%)$ & $7736(98.5 \%)$ & $384(15.1 \%)$ & $2546(98.6 \%)$ \\
\hline
\end{tabular}

$\mathrm{SD}$, standard deviation; $\mathrm{HR}$, heart rate; $\mathrm{SBP}$, systolic blood pressure; $\mathrm{SpO}_{2}$, peripheral oxygen saturation.

a Obesity is defined as medical history or body mass index $\geq 30 \mathrm{~kg} / \mathrm{m}^{2}$.

b History of cerebrovascular disease, peripheral arterial disease, myocardial infarction, angina pectoris, heart failure or atrial fibrillation.

c Chronic obstructive pulmonary disease, obstructive sleep apnoea/hypopnoea syndrome and asthma.

${ }^{\mathrm{d}}$ History of serum creatinine level $>3 \mathrm{mg} / \mathrm{dL}$ or history of dialysis.

e History of solid tumour, leukaemia or lymphoma.

${ }^{\mathrm{f}}$ History of autoimmune diseases, solid-organ transplant recipients, HIV infection or previous immunosuppressive treatment including systemic steroids.

$g$ Moderate or severe dependency for activities of daily living (Barthel index score $<60$ ).

h Temperature $\geq 38^{\circ} \mathrm{C}$ or history of fever.

\section{Sensitivity analysis}

We carried out a complete-case analysis selecting as development cohort the 5513 patients with complete data on the 21 potential predictors and the outcome. The resulting model had the same predictors as the PRIORITY model with apparent C-statistic 0.813 (95\%CI $0.800,0.826)$ and calibration slope 0.993 (95\%CI 0.986, 0.997). Next, we fitted a new model with no restriction in the maximum number of variables, resulting in a model which added sex, diabetes mellitus, malignancy, immunocompromised status, pulmonary rales, and heart rate cubed to the predictors in the PRIORITY model. C-statistic was 0.831 (95\%CI 0.821, 0.842) and slope 0.990 (95\%CI 0.986, 0.996). Likewise, we fitted an alternative model using linear continuous predictors instead of non-linear terms, which included sex but excluded the systolic blood pressure. C-statistic was $0.823(95 \% \mathrm{CI}$ $0.812,0.833)$ and slope 0.994 (95\%CI 0.988, 0.999).

\section{Discussion}

We developed and validated a new clinical risk model to predict COVID-19 critical illness based on nine simple clinical features easily available on initial assessment, which would be useful in resource-limited or out-of-hospital settings without access to other complementary tests. The model was well calibrated, had good discrimination, and performed robustly in an external validation cohort. Moreover, it showed a potential clinical benefit in a variety
Table 2

Multivariate logistic regression of critical illness prediction in coronavirus 2019 (COVID-19)

\begin{tabular}{lll}
\hline Predictors & Odds ratio & $95 \% \mathrm{CI}$ \\
\hline${\text { (Age } / 100)^{2} \text { Age in years }}^{\mathrm{a}}$ & 14.339 & $10.054,20.532$ \\
Cardiovascular disease $_{\text {Severe chronic kidney disease }}$ & 1.372 & $1.195,1.573$ \\
Dyspnoea & 1.797 & $1.433,2.252$ \\
$1 /(\mathrm{SBP} / 100)^{2} \mathrm{SBP}$ in $\mathrm{mmHg}^{\mathrm{a}}$ & 1.655 & $1.451,1.891$ \\
$\mathrm{Tachypnoea}(>20 \text { breaths } / \mathrm{min})_{\mathrm{SpO}_{2} \leq 93 \% \text { or oxygen requirement }}^{2.326}$ & $1.837,2.951$ \\
Confusion $_{\text {Dependency (moderate or severe) }}$ & 2.487 & $2.192,2.824$ \\
\hline
\end{tabular}

Predictors in the PRIORITY model retained after LASSO feature selection. Model coefficients were derived from a multivariate logistic regression, and presented as odds ratios (ORs) and 95\% confidence intervals (95\%CIs). Variables entered into the LASSO feature selection process were: age as a squared term, sex, ethnicity, smoking history, obesity, hypertension, diabetes mellitus, cardiovascular disease, pulmonary diseases, severe chronic kidney disease, malignancy, immunocompromised status, dependency, fever, dyspnoea, systolic blood pressure (SBP) as the inverse of a quadratic term, heart rate (HR) as a cubic term, tachypnoea, peripheral oxygen saturation $\left(\mathrm{SpO}_{2}\right) \leq 93 \%$ on room air or oxygen requirement at presentation, pulmonary rales, and confusion. All predictors were coded as binary variables ( 1 when present and 0 when absent) except for age (years), SBP ( $\mathrm{mmHg}$ ) and HR (bpm).

a Continuous predictors modelled as fractional polynomial terms, including rescaling when the range of values of the predictor was reasonably large. As interpretability of the effect of non-linear continuous predictors can be difficult, linear local approximations of ORs for 10-unit variations are provided at selected values. ORs for age (10-year increments): OR $(50 / 40)=1.271$; OR $(70 / 60)=1.414$; OR $(90 / 80)=1.573$. ORs for SBP $(10-\mathrm{mmHg}$ decreases $)$ : OR $(110 / 120)=1.118$; OR $(90 / 100)=1.219$; OR $(70 / 80)=1.497$. Approximated ORs are provided for illustrative purposes only and were not used for making predictions. 
a

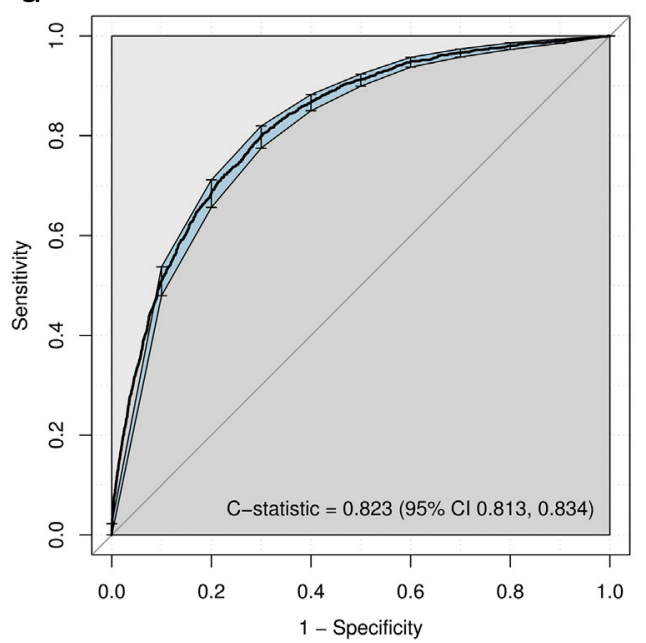

b

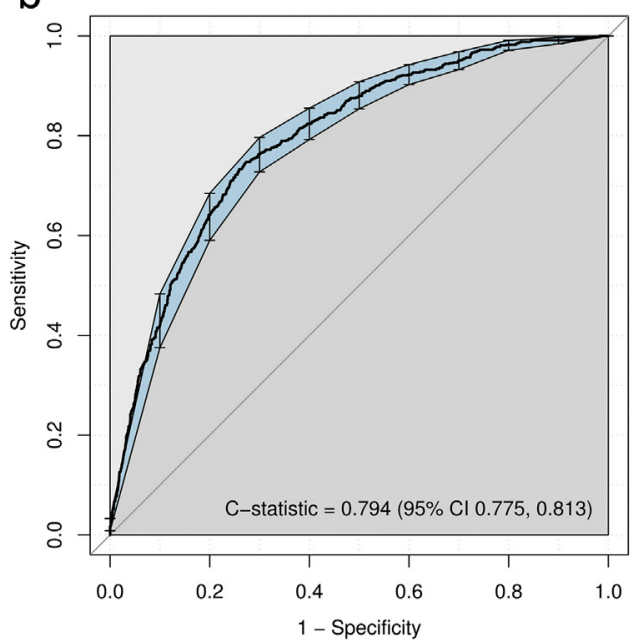

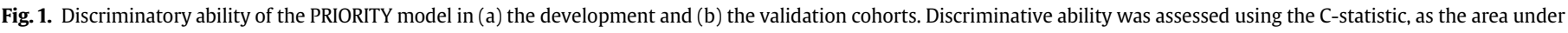
the receiver operating characteristic curve, with 95\% confidence intervals (CIs) computed with 1000 bootstrap replicates.

of scenarios covering different healthcare situations over a range of threshold probabilities for critical illness. The web-based calculator can facilitate its immediate application for frontline clinicians.

Previously, an external validation of 22 prognostic models showed that none of the multivariate models offered incremental value for patient stratification compared to oxygen saturation or age [15]. In this regard, the PRIORITY model showed higher discriminative ability and net benefit than age and/or oxygen saturation. Additionally, despite its simplicity, our model had a performance similar to previously published prognostic tools including laboratory and imaging tests [9-16].

It is worth noting that the PRIORITY model could be applied in triage, using easily measurable variables available in settings without access to laboratory or radiology tests, identifying highrisk patients for referral to hospital. This model could be useful for supporting clinical management decisions over a range of risk thresholds for critical illness which could be considered as relevant in clinical practice. The choice of thresholds will vary across different regions, according to changing epidemiological situations and availability of health resources. For example, under pandemic peak pressure or low-resource healthcare systems, policy-makers may consider a cut-off point up to $20 \%$, a threshold that will be associated with higher reduction in unnecessary critical care admissions. However, at low risk of overwhelming the critical care capacity, a lower threshold may be considered at the expense of unnecessary referrals. We recommend objectively defining specific cut-off points considering the circumstances and the availability of health resources.

This study has several methodological strengths maximizing internal and external validity [23]. To the best of our knowledge,

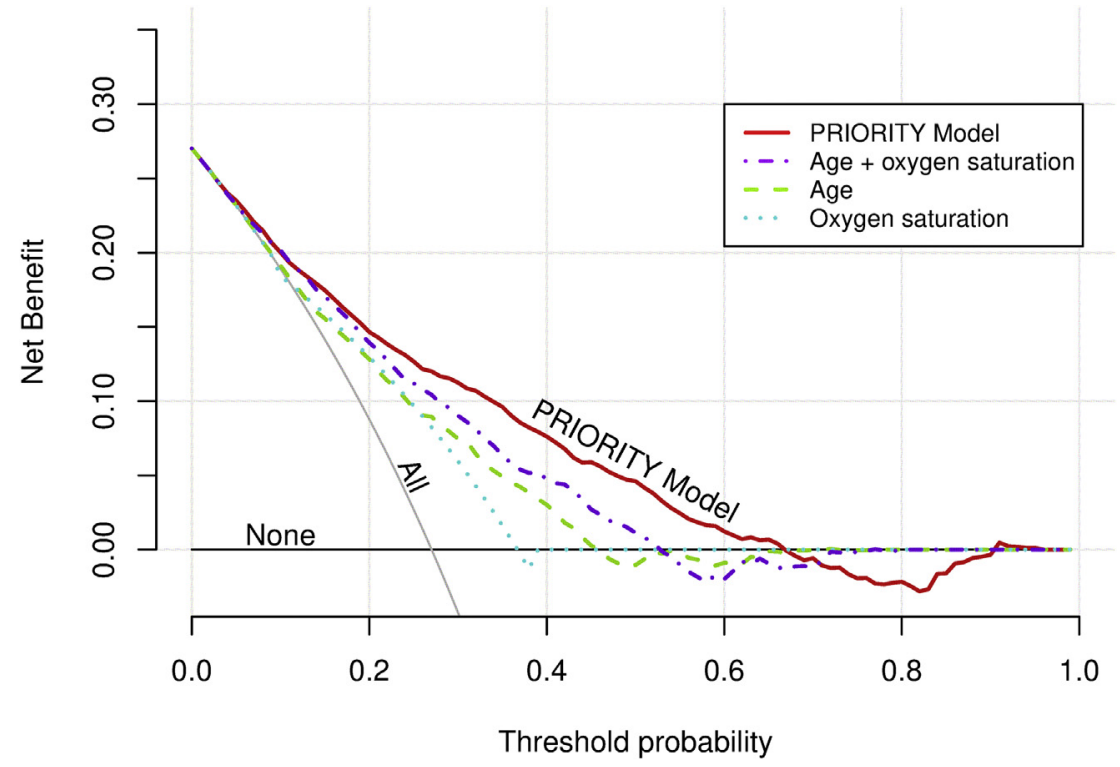

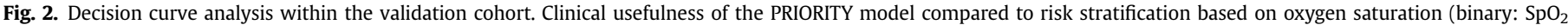

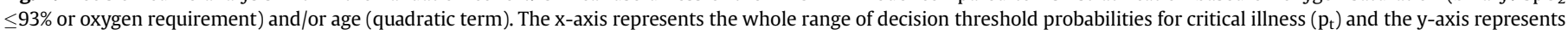
the net benefit (NB). NB calculated as: True positives/N - (false positives/N)*( $\mathrm{p}_{t} /\left(1-\mathrm{p}_{\mathrm{t}}\right)$ ), with $\mathrm{N}$ total sample size. 
this is the first generalizable COVID-19 predictive model built with simple clinical information (excluding imaging and laboratory data). We developed and validated the model in a large multicentre national cohort. The methodology was rigorous, avoiding datadriven predictor selection and biases that have affected previous studies [14]. The practical application of the model was maximized by forging an agreement among an expert panel on key issues. Moreover, the model was validated in a separate cohort of patients admitted in smaller hospitals, showing transportability to a setting with a different level of healthcare [21,22].

The strengths of our findings should be interpreted in light of some limitations. First, although we carefully selected easily available clinical and demographic variables, the data were collected at the time of hospital admission, which represents an important selection bias that would require further studies in an outpatient setting. Second, it could be suggested that, taking into account the situation of healthcare pressure, data quality may be affected. In this regard, it is notable that in this study there were relatively few missing data and we used imputation to reduce their impact. Third, since the COVID-19 pandemic has demonstrated significant differences between countries and time periods, it could affect the applicability of the model to other settings. However, we considered this early pandemic period in Spain to reflect a scenario with an overwhelmed healthcare system, where our predictive model could be particularly useful. Nevertheless, further studies introducing factors such as viral strains, healthcare system actions, new treatments, or vaccination could improve the applicability of the PRIORITY model. Finally, even though we compared the net benefit of using the model with discrimination based on oxygen saturation and/or age, its real clinical usefulness would require comparison with the best existing scores or the clinician's decision.

In summary, we have developed and validated a new prediction model, called PRIORITY, to estimate the risk of critical illness in patients with COVID-19 based on nine clinical variables easily measurable in resource-limited or out-of-hospital settings. The study could provide underpinning evidence to inform decisionmaking in health systems under pandemic pressure.

\section{Author contributions}

MML, AVN and MF planned and conceived the study and analysed and interpreted the data. MML, AVN, MF and LM wrote the original draft of the manuscript. MRR, SLG, FAF, JLBP, JAVN, ECM, ACEA, SJFC, JLA, PMPF, AP, AMAS, ASA, BGL, JLP, JSC, PCP, GMGG, JMNC, JMCR and RGH contributed to reading and approving the final version of the manuscript. MML and AVN are joint first authors. The corresponding author attests that all listed authors meet authorship criteria and that no others meeting the criteria have been omitted. LM is the guarantor.

\section{Transparency declaration}

The corresponding author affirms that the manuscript is an honest, accurate, and transparent account of the study being reported; that no important aspects of the study have been omitted; and that any discrepancies from the study as planned have been explained. The authors have no conflicts of interest to declare. No funding was received for this work.

\section{Acknowledgements}

We gratefully acknowledge all the investigators who participate in the SEMI-COVID-19 Registry (listed at Supplementary Appendix
S1), especially those from Hospital Universitario Ramón y Cajal (Luis F. Abrego-Vaca, Ana Andreu-Arnanz, Octavio A. Arce-García, Marta Bajo-González, Pablo Borque-Sanz, Alberto Cózar-Llistó, Beatriz Del Hoyo-Cuenda, Alejandra Gamboa-Osorio, Isabel GarcíaSánchez, Óscar A. López-Cisneros, Borja Merino-Ortiz, Elisa RieraGonzález, Jimena Rey-García, Cristina Sánchez-Díaz, Grisell StaritaFajardo, Cecilia Suárez-Carantoña, Svetlana Zhilina Zhilina). We especially thank our colleagues at Hospital Universitario Ramon y Cajal, IRYCIS: Nuria Bara Ledesma, Andrés González García and José Luis Calleja López (Internal Medicine Dept), Javier Zamora and Borja M. Fernandez-Felix (Clinical Biostatistics Unit), and Javier Soto Pérez-Olivares (Radiology Dept) for their valuable contributions to the review of the manuscript. The authors would also like to gratefully acknowledge Professor Khalid S. Khan, Distinguished Investigator at the University of Granada, Spain, for his support and advice on the manuscript. Finally, we also thank the SEMI-COVID19 Registry Coordinating Center, S\&H Medical Science Service, for their quality control data, logistic and administrative support.

\section{Dissemination to participants and related patient and public communities}

Results of this study have been made available to the public through an open access preprint posted to MedRxiv (https://doi. org/10.1101/2020.11.27.20237966). The Spanish Society of Internal Medicine (SEMI) shares the results of the studies derived from the SEMI-COVID-19 Registry through its public-facing website (https:// www.fesemi.org/investigacion/proyectos/registro-semi-covid-19) and its twitter account (@Sociedad_SEMI).

\section{Appendix A. Supplementary data}

Supplementary data to this article can be found online at https://doi.org/10.1016/j.cmi.2021.07.006.

\section{Data sharing}

The data that support the findings of this study are available on request from the SEMI-COVID-19 Scientific Committee and the Registry Coordinating Center.

\section{References}

[1] Docherty AB, Harrison EM, Green CA, Hardwick HE, Pius R, Norman L, et al. Features of 20133 UK patients in hospital with covid-19 using the ISARIC WHO Clinical Characterisation Protocol: prospective observational cohort study. BMJ 2020;369:m1985.

[2] Guan W, Ni Z, Hu Y, Liang W, Ou C, He J et al. Clinical characteristics of coronavirus disease 2019 in China. N Engl J Med;382:1708-1720.

[3] WHO. COVID-19 situation reports. 2020. https://www.who.int/emergencies/ diseases/novel-coronavirus-2019/situation-reports. [Accessed 24 May 2021].

[4] Ministerio De Sanidad, Consumo y Bienestar Social. Enfermedad por el coronavirus (COVID-19). 2020 (in Spanish), https://www.mscbs.gob.es/en/ profesionales/saludPublica/ccayes/alertasActual/nCov/documentos/ Actualizacion_288_COVID-19.pdf. [Accessed 28 May 2021].

[5] Wang D, Hu B, Hu C, Zhu F, Liu X, Zhang J, et al. Clinical characteristics of 138 hospitalized patients with 2019 novel coronavirus-infected pneumonia in Wuhan, China. JAMA 2020;323:1061-9.

[6] Zhou F, Yu T, Du R, Fan G, Liu Y, Liu Z, et al. Clinical course and risk factors for mortality of adult inpatients with COVID-19 in Wuhan, China: a retrospective cohort study. Lancet 2020;395:1054-62.

[7] Richardson S, Hirsch JS, Narasimhan M, Crawford JM, McGinn T, Davidson KW, et al. Presenting characteristics, comorbidities, and outcomes among 5700 patients hospitalized with COVID-19 in the New York City area. JAMA 2020;323:2052-9.

[8] Berenguer J, Ryan P, Rodríguez-Baño J, Jarrín I, Carratalà J, Pachón J, et al. Characteristics and predictors of death among 4,035 consecutively hospitalized patients with COVID-19 in Spain. Clin Microbiol Infect 2020;26:1525-36. 
[9] Fumagalli C, Rozzini R, Vannini M, Coccia F, Cesaroni G, Mazzeo F, et al. Clinical risk score to predict in-hospital mortality in COVID-19 patients: a retrospective cohort study. BMJ Open 2020;10:e040729.

[10] Liang W, Liang H, Ou L, Chen B, Chen A, Li C, et al. Development and validation of a clinical risk score to predict the occurrence of critical illness in hospitalized patients with COVID-19. JAMA Intern Med 2020;180:1081-9.

[11] Ji D, Zhang D, Xu J, Chen Z, Yang T, Zhao P, et al. Prediction for progression risk in patients with COVID-19 pneumonia: the CALL Score. Clin Infect Dis 2020;71:1393-9.

[12] Knight SR, Ho A, Pius R, Buchan I, Carson G, Drake TM, et al. Risk stratification of patients admitted to hospital with covid-19 using the ISARIC WHO Clinical Characterisation Protocol: development and validation of the 4C Mortality Score. BMJ 2020;370:m3339.

[13] Bartoletti M, Giannella M, Scudeller L, Tedeschi S, Rinaldi M, Bussini L, et al. Development and validation of a prediction model for severe respiratory failure in hospitalized patients with SARS-Cov-2 infection: a multicenter cohort study (PREDI-CO study). Clin Microbiol Infect 2020;26: 1545-53.

[14] Wynants L, Van Calster B, Collins GS, Riley RD, Heinze G, Schuit E, et al. Prediction models for diagnosis and prognosis of COVID-19 infection: systematic review and critical appraisal. BMJ 2020;369:m1328.

[15] Gupta RK, Marks M, Samuels T, Luintel A, Rampling T, Chowdhury H, et al. Systematic evaluation and external validation of 22 prognostic models among hospitalised adults with COVID-19: an observational cohort study. Eur Respir J 2020;56:2003498.

[16] Yadaw AS, Li YC, Bose S, Iyengar R, Bunyavanich S, Pandey G. Clinical features of COVID-19 mortality: development and validation of a clinical prediction model. Lancet Digit Health 2020;2:e516-25.

[17] Ajayi-Obe EK, Lodi E, Alkali AS, Galbati M, Rooney C, Mannoni B, et al. Prognostic scores for use in African meningococcal epidemics. Bull World Health Organ 1998;76:149-52.

[18] Bassat Q Lanaspa M, Machevo S, O'Callaghan-Gordo C, Madrid L, Nhampossa $T$, et al. Hypoxaemia in Mozambican children $<5$ years of age admitted to hospital with clinical severe pneumonia: clinical features and performance of predictor models. Trop Med Int Health 2016;21:1147-56.
[19] Lanaspa M, Valim C, Acacio S, Almendinger K, Ahmad R, Wiegand R, et al. High reliability in respiratory rate assessment in children with respiratory symptomatology in a rural area in Mozambique. J Trop Pediatr 2014;60:93-8.

[20] Casas Rojo JM, Antón Santos JM, Nuñez-Cortés J, Lumbreras-Bermejo C Ramos-Rincón JM, Roy-Vallejo E, et al. Clinical characteristics of patients hospitalized with COVID-19 in Spain: results from the SEMI-COVID-19 Network. Rev Clin Esp 2020;220:480-94.

[21] Justice AC, Covinsky KE, Berlin JA. Assessing the generalizability of prognostic information. Ann Intern Med 1999:130:515-24.

[22] Steyerberg EW, Vergouwe Y. Towards better clinical prediction models: seven steps for development and an $\mathrm{ABCD}$ for validation. Eur Heart 2014;35:1925-31.

[23] Ministerio de Sanidad, Consumo y Bienestar Social. Certificates of discharge of the national health system register. 2020. CMBD. State Regulation (in Spanish), https://www.mscbs.gob.es/en/estadEstudios/estadisticas/cmbd.htm. [Accessed 25 May 2021].

[24] Metlay JP, Waterer GW, Long AC, Anzueto A, Brozek J, Crothers K, et al. Diagnosis and treatment of adults with community-acquired pneumonia. An officia clinical practice guideline of the American Thoracic Society and Infectious Diseases Society of America. Am J Respir Crit Care Med 2019;200:e45-67.

[25] Evangelidis, Nicole Tong A, Howell M, Teixeira-Pinto A, Elliott JH, Azevedo LC, et al. International survey to establish prioritized outcomes for trials in people with coronavirus disease 2019. Crit Care Med 2020;48:1612-21.

[26] Fitch K, Bernstein S, Aguilar MD, Burnand B, LaCalle JR, Lazaro P, et al. The RAND/UCLA appropriateness method user's manual. 1st ed. Santa Monica, CA: RAND Corporation; 2001.

[27] Steyerberg EW. Clinical prediction models: a practical approach to development, validation and updating. 2nd ed. New York: Springer; 2019.

[28] Royston P, Altman DG. Regression using fractional polynomials of continuous covariates: parsimonious parametric modelling. Appl Stat 1994;43: 429-67.

[29] Hastie T, Tibshirani R, Friedman JH. The elements of statistical learning: data mining, inference, and prediction. 2nd ed. New York: Springer; 2009.

[30] Vickers AJ, Elkin EB. Decision curve analysis: a novel method for evaluating prediction models. Med Decis Making 2006;26:565-74. 